\title{
Prototype HNX/TIGERISS Silicon Strip Detector Response to Nuclei Measured in a Lead Test Beam
}

\author{
John F. Krizmanic* \\ NASA/GSFC/University of Maryland, Baltimore County \\ E-mail: John.F.Krizmanic@nasa.gov \\ John W. Mitchell \\ $N A S A / G S F C$ \\ E-mail: John.W.Mitchell@nasa.gov
}

Makoto Sasaki

NASA/GSFC/University of Maryland, College Park

E-mail: Makoto.Sasaki@nasa.gov

for the HNX \& TIGERISS Collaborations and CERN Test Beam Team ${ }^{\dagger}$

\begin{abstract}
The response to primary and fragmented lead test beams measured by prototype silicon strip detectors, designed for use in the Heavy Nuclei eXplorer (HNX) and the Trans-Iron Galactic Element Recorder on the International Space Station (TIGERISS) experiments, was evaluated in a CERN test beam in Nov - Dec 2016. The $500 \mu \mathrm{m}$ thick, single-sided prototype silicon detectors have 32 DC-coupled strips with $3 \mathrm{~mm}$ pitch on the junction side with an approximate $10 \times 10$ $\mathrm{cm}^{2}$ active area. Three different types of electronics were employed to readout the prototype detectors in order to test different read out methodologies, and in each case both the junction and ohmic sides were read out simultaneously. During the test beam runs, the prototype detectors were situated between planar silicon detectors, which provided a comparison of the measured response of each detector. The combined data set shows excellent charge resolution and clearly resolved elemental peaks from carbon $(Z=6)$ through lead $(Z=82)$. A description of the test beam experiments and a discussion of the results are reported.
\end{abstract}

35th International Cosmic Ray Conference - ICRC2017

10-20 July, 2017

Bexco, Busan, Korea

\footnotetext{
* Speaker.

$\dagger$ John Mitchell, Terri Brandt, John Krizmanic, Jason Link, Sara Mitchell, Kenichi Sakai, \& Makoto Sasaki (NASA/GSFC)
} 


\section{Introduction}

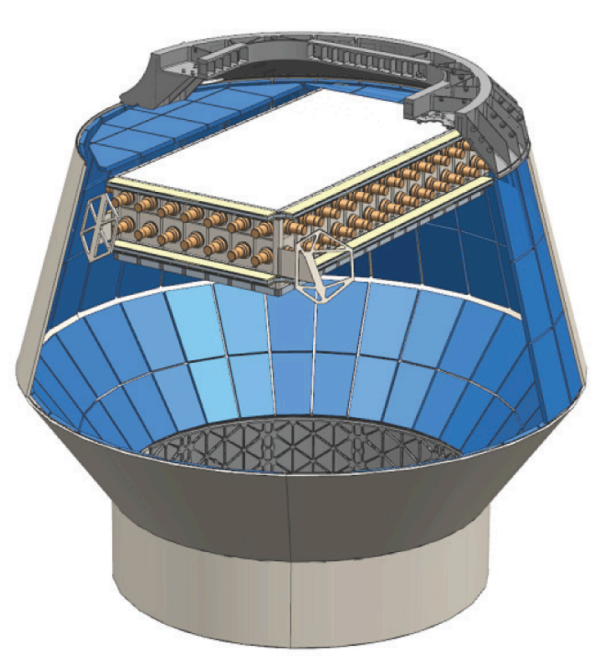

Figure 1: A schematic of the ECCO and CosmicTIGER HNX instruments in a pressurized DragonLab capsule. The capsule is returned to Earth in order to recover and process the ECCO BP-1 tiles.
The proposed Heavy Nuclei eXplorer (HNX) mission $[1,2,3]$ is designed to measure the individual elemental abundances of cosmic rays, from carbon through curium, in a nominal two year mission. HNX is comprised of two instruments, the Extremelyheavy Cosmic-ray Composition Observer (ECCO) and the Cosmic-ray Trans-Iron Galactic Element Recorder (CosmicTIGER). A schematic of the instruments in a DragonLab capsule is shown in Figure 1. ECCO employs $\sim 21 \mathrm{~m}^{2}$ of barium phoshate (BP-1) glass tiles to record individual $\mathrm{CR}$ nuclei with $Z \geq 70$ with high sensitivity through the actinides. ECCO, based on the Trek experiment [4], requires the BP-1 tiles to be returned to the Earth for processing. CosmicTIGER is an electronic instrument with sufficient dynamic range to measure cosmic-ray (CR) nuclei from carbon through curium. Thus CosmicTIGER has a large measurement overlap with ECCO, especially in the $70 \leq Z \leq 82$ region where event statistics are sufficiently large. Above $Z=82$, statistics will be limited for CosmicTIGER in a 2 year flight. CosmicTIGER has an area of $2 \mathrm{~m}^{2}$ and utilizes three detector subsystems: silicon strip detector (SSD) arrays, an acrylic $(\mathrm{n}=1.5)$ Cherenkov detector, and an aerogel $(n=1.04)$ Cherenkov detector. The Cherenkov detectors provide measurement of charge and velocity with two different $\mathrm{CR}$ energy thresholds. The silicon detectors provide charge measurements with excellent charge resolution and also provide measurement of individual nuclei trajectories. These allow the identification of CR nuclei at the individual element level via the use of the $d E / d x$ versus Cherenkov and Cherenkov versus Cherenkov techniques.

CosmicTIGER uses 4 planes of SSDs, two above

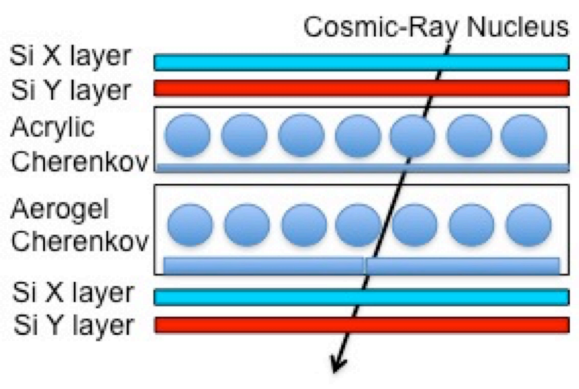

Figure 2: A schematic of the CosmicTIGER instrument illustrating the silicon strip detector and Cherenkov subsystems. and two below the Cherenkov detectors, in an alternating $\mathrm{x}-\mathrm{y}$ arrangement. A schematic is shown in Figure 2. Each plane is comprised of an array of $10 \times 20$ SSDs each with $\sim 10 \times 10 \mathrm{~cm}^{2}$ active area. Each SSD is a $500 \mu \mathrm{m}$ thick, single-sided DC-coupled silicon strip detector with 32 channels with $3 \mathrm{~mm}$ pitch on the junction side. Ten SSDs will be daisy-chained together to form ladders, with each ladder being read out by PHASIC front-end electronics [5]. The ohmic side of each SSD will be read out by a discrete chargepreamplifier chain to provide a redundant charge measurement and event triggering signals. The anticipated charge resolution of the SSD subsystem is $\sigma_{Z}<0.25$ 
up to $\mathrm{Z}=96$. TIGERISS [1] is a smaller version of the CosmicTIGER instrument, with TIGERISS developed for attachment to the International Space Station.

\section{HNX/TIGERISS Prototype Silicon Strip Detectors}

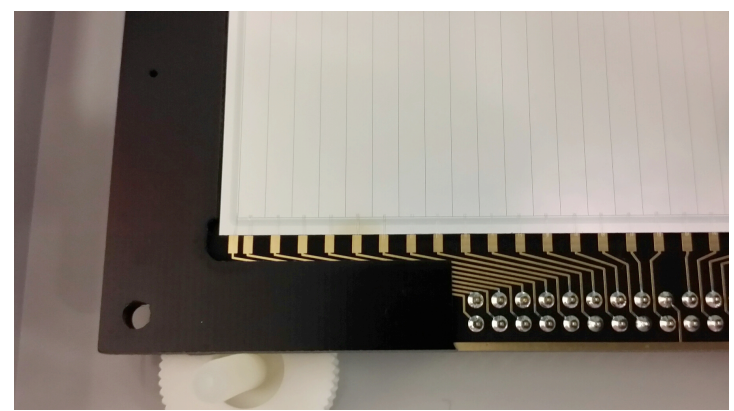

Figure 3: A picture of the strip side of a mounted prototype SSD.

Five prototype HNX/TIGERISS SSD detectors were obtained from Micron Semiconductors LTD. Each single-sided, DCcoupled detector has an active area of $96 \times 96$ $\mathrm{mm}^{2}$ and range in thickness from 502 to $509 \mu \mathrm{m}$. The SSDs have 32 junction-sided strips with a pitch of $3 \mathrm{~mm}$. Each detector was mounted to a window pane circuit board. The SSD strip, guard ring, and field plate channels were wire bonded to pads on the board at each end of the detector, and the traces connected to a two row pin connector.

A picture of the corner of a strip side of a mounted SSD is shown in Figure 3. The detectors have a depletion voltage of $\leq 32 \mathrm{~V}$ and have a total leakage current (32 strips + guard ring) of $\sim 100$ $\mathrm{nA}$ at $100 \mathrm{~V}$. The depletion voltage and total leakage current for each detector were validated in the laboratory at GSFC via CV and IV measurements at $\sim 22^{\circ} \mathrm{C}$. The distribution of leakage currents per strip for each detector showed small variation with a few channels having $\sim 1 \mathrm{nA}$ enhanced current/strip. Three detectors have a leakage current of $\sim 3 \mathrm{nA} / \mathrm{strip}$ at $V_{D E P}+30 \mathrm{~V}$ while two detectors have $\sim 1 \mathrm{nA} /$ strip at $V_{D E P}+30 \mathrm{~V}$. These two detectors were chosen to be tested in the CERN beam test.

\section{CERN Test Beam Configuration}

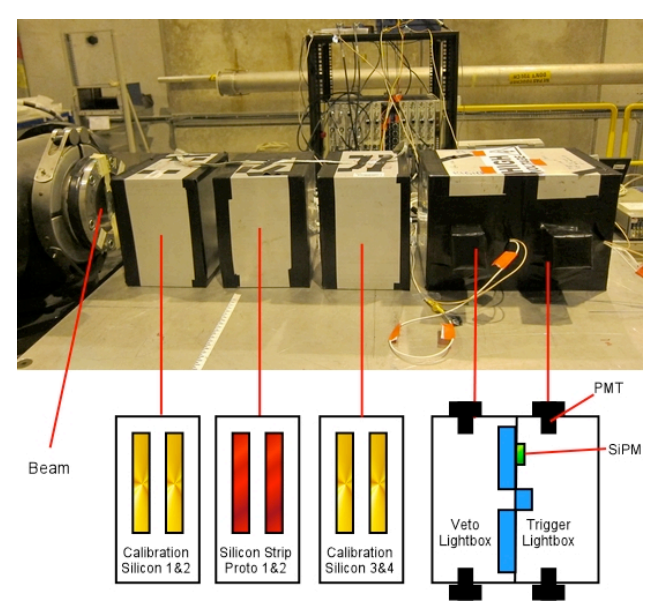

Figure 4: A annotated picture of the CERN test beam experimental setup.
The CERN beam test occurred November 30 , 2016 to December 6, 2016 in the H8A beamline in the North Area of CERN. The primary beam consisted of $\mathrm{Pb}$ nuclei with $30 \mathrm{GeV} /$ nucleon kinetic energy for the majority of the test, albeit the beam energy was changed to $150 \mathrm{GeV} /$ nucleon on December 5, 2016. An annotated picture of the experimental setup is shown in Figure 4. The two prototype SSD (pSSDs) detectors were mounted in a lightbox and placed between calibration silicon detectors (cSDs), with two upstream and two downstream of the pSSDs. The cSDs were $\sim 10 \times 10 \mathrm{~cm}^{2}, 500 \mu \mathrm{m}$ thick pad detectors with the junction and ohmic sides of each detector read out individually. The strips on each of the two 
pSSD detectors were electrically connected together, and the junction and ohmic sides were read out separately. Figure 5 shows the two pSSD detectors mounted in their lightbox. The cSDs and pSSDs were mounted at a 10 degree angle with respect to the beam direction in order to minimize any crystal channeling effects. The cSDs and pSSDs used charge preamplifiers coupled to shaping amplifiers whose signals were then digitized by 12-bit ADCs via Lecroy 2259B peak-sensing ADC modules. The ADC modules were modified such that a voltage bias provided an ADC offset to provide some compensation for known non-linearity at the low ADC range. The cSDs used 4-channel custom preamplifier modules, based the RL-724 charge preamplifier (manufactured by Rel-Labs) with $C_{f}=33 \mathrm{pF}$, whose outputs were individually amplified by Canberra 2022 shaping amplifiers using $1 \mu$ s shaping times. This is denoted as $c$ SDreadout. There were three different configurations of the front-end electronics used for the readout of the pSSDs in this beam test, and these will be described below. The cSDs and pSSDs were biased at positive 60 volt depletion voltage on the ohmic sides during all tests. The guard rings and field plates of the pSSDs were grounded for all tests.

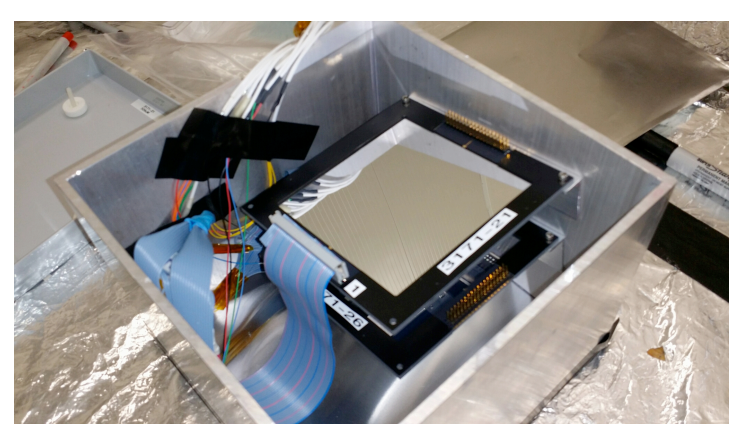

Figure 5: The mounting of the two pSSDs in their lightbox.

The test beam trigger used a 0.88 -inch diameter, 0.5-inch thick circular scintillator in a lightbox that was coupled to two PMTs. A 0.5 -inch thick, $5 \times 7$ in $^{2}$ veto scintillator with a 1-inch diameter circular hole that was aligned to the trigger scintillator was located in a separate light box, also coupled to 2 PMTs. The veto scintillator was located immediately in front of the trigger light box. The event trigger was formed by accepting a coincidence signal using the two trigger PMTs while requiring no veto signal defined by a coincidence signal from its two PMTs. Thus events were only accepted from the $\sim 1 \mathrm{~cm}$ diameter central beam. A SiPM was included in the trigger light box, and its beam response is reported in [6]. NIM and CAMAC electronics were used to process the signals and provide data to a controlling computer. A beam spill signal was used to define pedestal runs between beam spills.

For each unique experimental configuration, the experimental procedure first began with a lead primary run to adjust the gains of the amplifiers in order to get a $Z=82$ peak at an ADC value near 1900. Once the gains were set, a primary lead run was performed with sufficient statistics to measure the $Z=82$ peak with good accuracy. Lead fragment runs were then performed by placing a polyethylene target in the lead primary beam and selecting different $A / Z$ fragments in the beam. $A / Z$ settings of $2.4,2.2$, and 2.0 were used during this beam test, with each setting selecting different $Z$-ranges of fragments in the beam. The ensemble of the different $A / Z$ runs yielded charge measurements of individual nuclei from lead $(Z=82)$ down to a $Z=17$ with the nominal trigger threshold setting (this was lowered in the later runs to get the Z-threshold down to below $Z=6$ ). The charge measurement data from the cSDs ans pSDs were then used to determine residual non-linear effects in the electronics response, yielding a calibration function that maps the $\mathrm{ADC}$ measurements to the charge of each individual nuclei in units of atomic number, $\mathrm{Z}$. 


\subsection{Experimental Setup 1: Use of charge attenuation with a limited-range preamplifier}

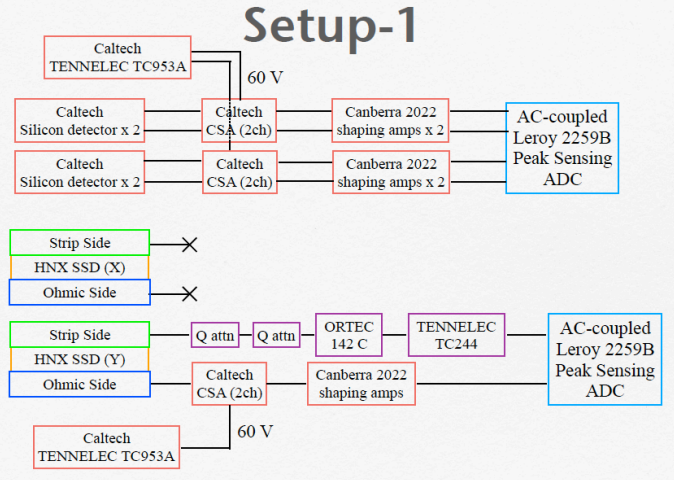

Figure 6: A schematic of experimental Setup 1.

The first experimental setup was designed to measure the performance of a system using charge attenuators with a charge preamplifier with limited dynamic range to read out the strip side on one of the pSSDs. A min-ionizing, normal incidence $Z=82 \mathrm{nu}$ cleus will generate $\sim 40 \mathrm{pC}$ in a $500 \mu \mathrm{m}$ thick silicon detector. If this amount of charge is to large for a specific preamplifier, charge attenuators can in principle reduce the amount of charge to bring the measurement within the range of the preamplifier. The purpose of this experiment was to demonstrate this capability with a pSSD, wich has a body capacitance of $\sim 2 \mathrm{nF}$.

Two charge attenuators $\left(Q_{\text {Atten }}\right)$ were fabricated using Pomona boxes. One with $C_{S}=10 \mathrm{nF}$ and $C_{P}=48 \mathrm{nF}$ had a $\times 5.8$ attenuation and another with $C_{S}=23 \mathrm{nF}$ and $C_{P}=51 \mathrm{nF}$ had a $\times 3.2$ attenuation. These were placed in between the strip-side connection of a pSSD and an Ortec 142c preamplifier $\left(C_{f}=2 \mathrm{pF}\right)$, which was attached to a Tennelec TC244 shaping amplifier that used a
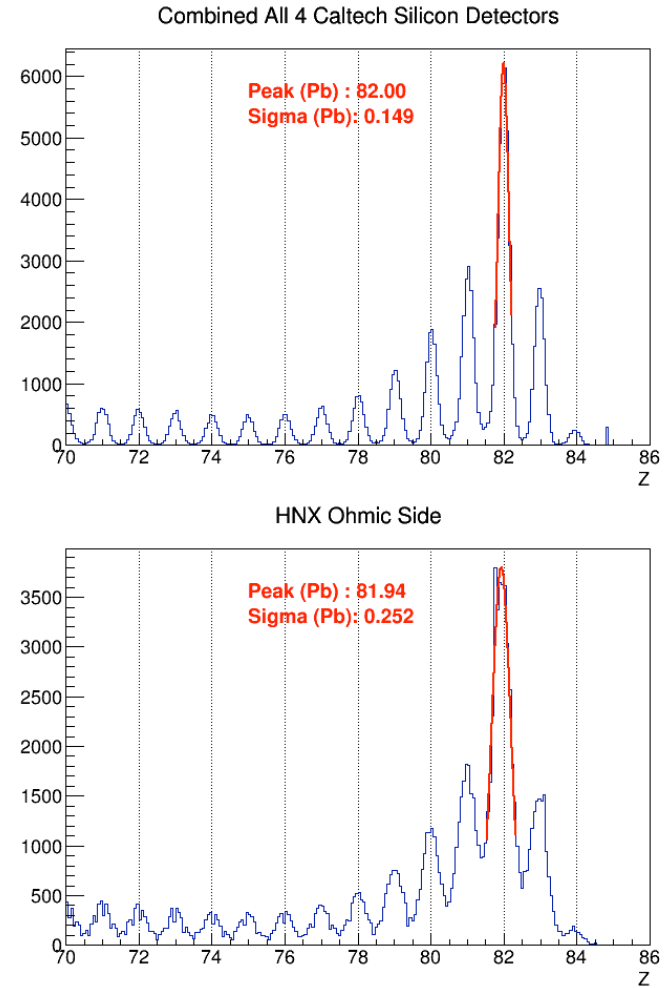

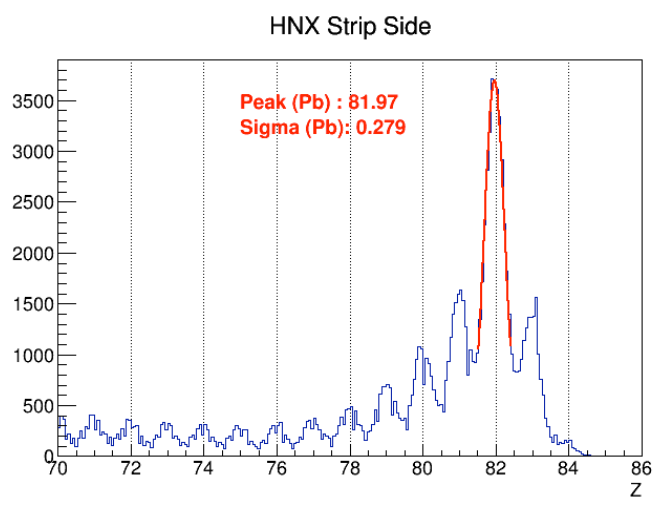

HNX Strip+Ohmic

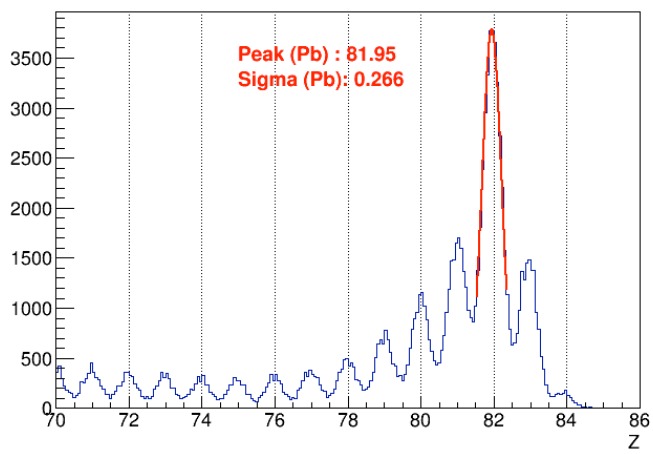

Figure 7: The measured response of the cSD detectors (combined) and the strip and ohmic side of a pSSD detector using $Q_{a t t e n}$ 's to bring the maximum measurement within the range of a Ortec 142c preamplifier. 
$1 \mu$ s integration time. The ohmic side of the pSSD used a spare cSDreadout channel to measure the charge from the ohmic side. A schematic of experimental Setup 1 is shown in Figure 6. The results comparing the combined response of the four cSDs detectors and strip and ohmic sides of a pSSD are shown in Figure 7 using data combined from both the primary lead and $A / Z=2.4$ fragment beam runs. The resolution of the $Z=82$ peak is similar for the pSSD ohmic side to that for the cSDs (taking into account the factor of 2 improvement in resolution obtained from combining 4 measurements), while the resolution for the strip side shows only a $\sim 11 \%$ worsening of the resolution. This implies that using $Q_{\text {Atten }}$ 's with a limited dynamic range preamplifier can be used to measure larger signals with comparable resolution to that obtained with a preamplifier with sufficient dynamic range for the measurement of large input charge.

\subsection{Experimental Setup 2: Calibration and prototype detectors response comparison using the same electronics}

The second experimental setup used the same front-end electronics for the cSDs and pSSDs. A schematic of experimental Setup 2 is shown in Figure 8. The limited number of cSDreadout channels required that two of the cSDs be not instrumented for readout (the furthest upstream and downstream cSDs). The response to the lead primary and fragment beams are shown in Figure 10. Also note that the threshold for the test beam trigger was lowered before the $A / Z=2.0$ run (last in the run sequence) in order to allow for the measurement of nuclei below $Z=6$. The results show excellent charge resolution through the range as well as excellent agreement with the charge resolution at lead $\left(\sigma_{Z} \leq 0.2\right)$ for both the calibration silicon detectors and the prototype silicon strip detectors. The charge resolution for the ohmic and strip sides of the pSSDs demonstrate complete agreement. Note that the lead primary results are shown in black and these events were used for the charge calibration. Also note that the $A / Z=2.4$ fragmented lead beam has fragments with $Z=82$ and $Z=83$. The reason the fraction of these to $Z=82$ is different than what is presented in Figure 7 is that the events from both the primary lead and the $A / Z=2.4$ fragment runs were added together to form Figure 7.

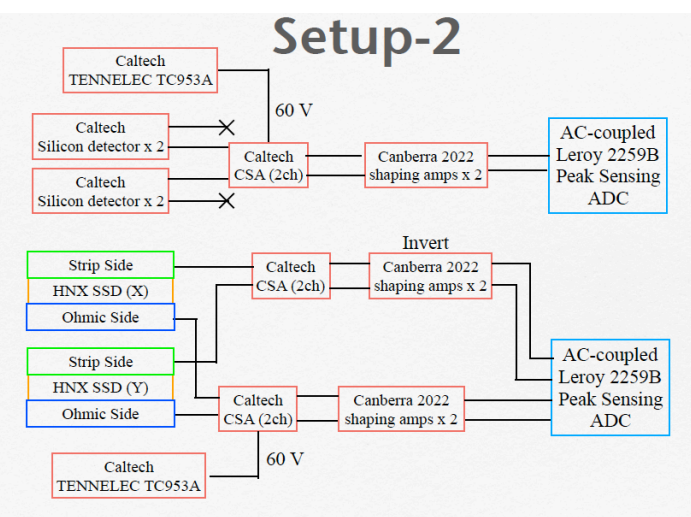

Figure 8: A schematic of experimental Setup 2.

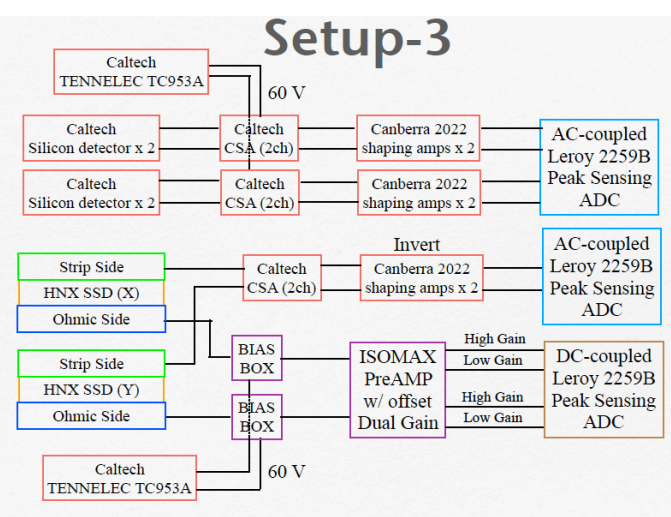

Figure 9: A schematic of experimental Setup 3.

\subsection{Experimental Setup 3: expanding the effective ADC range using ISOMAX preamplifiers}

The third experimental setup replaced the $c$ SDreadout on the pSSD ohmic sides with a dual- 

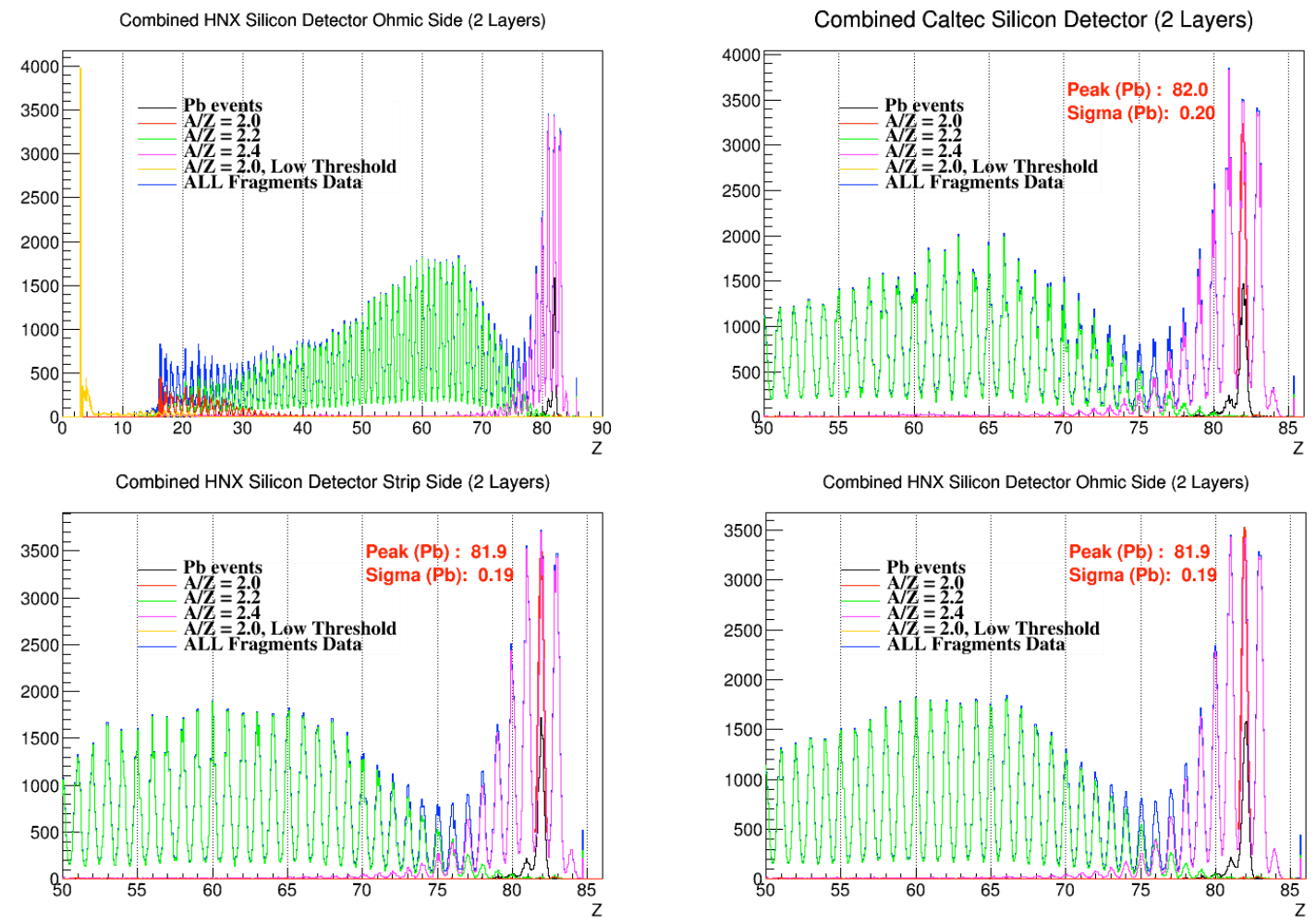

Figure 10: The measured response of the combined two pSSD ohmic side measurements (top left) for the combined results using a lead primary beam and fragments; combined results using two cSDS (top right) for $Z \geq 50$; combined results for the strip side using two pSSDs (bottom left) for $Z \geq 50$; and combined results for the ohmic side using two pSSDs (bottom left) for $Z \geq 50$. Note that the lead primary results are shown in black and these events were used for the charge calibration.

gain amplifier electronics used for the ISOMAX [7] experiment. The preamplifier for each channel is an AMPTEK A203 (with $C_{f}=58 \mathrm{pF}$ ) followed by dual low $(\times 1)$ and high $(\times 4)$ gain output. This effectively increases the ADC span by 2 bits for the ohmic side measurement and allows for the more precise measurement of nuclei, especially for $Z<10$. Bias boxes using $50 \mathrm{nF}$ coupling capacitors and $R_{\text {Bias }}=10 \mathrm{M} \Omega$ were used to bias the pSSDs from the ohmic sides. A schematic of experimental Setup 3 is shown in Figure 9. Data were taken using $30 \mathrm{GeV} /$ nuc lead primary and $A / Z=2.0$ fragment beams.

The lead beam energy was changed to $150 \mathrm{GeV} /$ nuc on the final days of testing. After a lead primary calibration run, the pSSD detector were rotated by an additional 20 degrees to perform measurements at an angle 30 degrees from normal incidence. Data was taken using the 150 $\mathrm{GeV} /$ nuc lead primary and $A / Z=2.0$ fragments. The data from this experimental setup was corrected using the calibrations obtained from Setup 2, and the combined results for Setup 2 and 3 are shown in Figure 11. The results show excellent charge resolution from carbon $(Z=6)$ through lead $(Z=82)$.

\section{Discussion}

The results of this beam test of this beam test show that the HNX/TIGERISS prototype sil- 


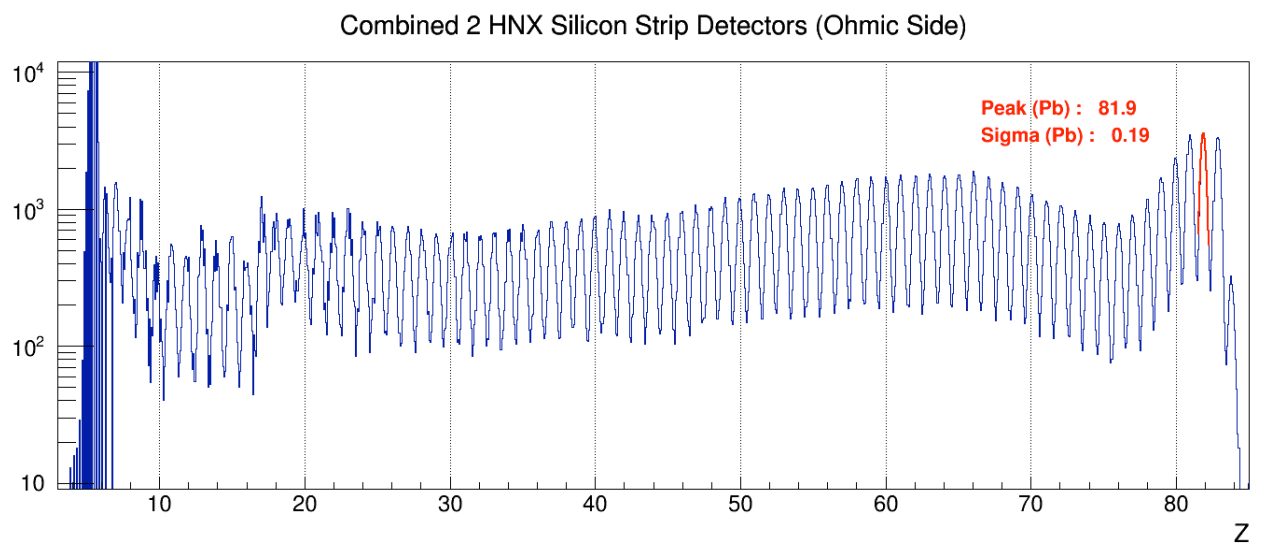

Figure 11: Combined results of the ohmic side measurement of two pSSDs for experimental Setup2 and Setup3 showing the individual nuclei resolution from carbon through lead.

icon strip detectors can measure nuclei from carbon through lead with superb charge resolution, $\sigma_{Z}<0.2$ at $Z=82$. This resolution was measured simultaneously from both the strip (junction) side as well as the ohmic side of the prototype SSDs (pSSDs). These results demonstrate the performance needed for the HNX and TIGERISS experiments and is anticipated to improve for the flight electronics. The pSSD resolution matches exceptionally well to $500 \mu \mathrm{m}$-thick silicon pad detectors used in this beam test for comparison and calibration. The results from the experimental setup using charge attenuators demonstrate that this technique can effectively be used with a preamplifier with limited charge measurement dynamic range. The modest increase in charge resolution observed $(\sim 11 \%)$ may be due to the inherent performance of the Ortec 142c preamplifier as compared to the Rel-Labs amplifiers that was used to make the comparison. This result implies that charge dividers could potentially be used to provide split inputs to different preamplifier-amplifier channels.

\section{Acknowledgements}

We thank Mark Wiedenbeck (CalTech/SRL/JPL) for the use of the silicon calibration detectors, the Rel-Labs preamplifier electronics, and the Canberra shaping amplifiers used in this beam test. We also thank Marco Ricci (INFN/Frascati) for help in obtaining electronics from the CERN electronics pool. Finally, we thank CERN for the opportunity to perform this beam test.

\section{References}

[1] J.W. Mitchell, these proceedings.

[2] T. Hams, et al., Proc of the $34^{\text {th }}$ ICRC (The Hague), PoS(ICRC2015)038 (2015)

[3] W.R. Binns et al., Proc of the $31^{\text {st }}$ ICRC (Łódź), Paper 0441 (2009)

[4] B.A. Weaver et al., NIMB, Vol 145, 3, p. 409-428 (1998)

[5] R.A. Mewaldt et al., Space Sci. Rev., Vol 136, 1-4, p. 285-362 (2008)

[6] J. Link et al., these proceedings.

[7] M. Hof et al., NIMA, Vol 454, 1, p. 180-185. (2000) 